\title{
L'impact de la coopération culturelle française sur la langue
}

\author{
Yaqdhan M-S ALASSAF \\ University of Mosul.Almajmoa, 41002, Mosul, Ninavah, Iraq \\ Yaqdhan_alassaf@uomosul.edu.iq
}

\section{Résumé}

Cette revue s'intéresse à la coopération culturelle et scientifique entre l'Irak et la France. Grâce à cette coopération, le Gouvernement français a mis en place un programme de bourses pour le master et le doctorat dans divers domaines. En ce qui concerne l'enseignement du français comme deuxième langue étrangère, le soutien inclut des stages de formation (2 à 4 semaines) offerts aux enseignants du français pour les aider à être informés du développement dans l'enseignement des langues. En outre, des manuels enseignés dans les départements du français de cinq universités irakiennes ont également été fournis grâce à cette coopération. Récemment, en octobre 2017, le Gouvernement français a confirmé sa volonté et son engagement à participer à la réhabilitation des bâtiments universitaires, à la redynamisation linguistique et de l'enseignement supérieur en Irak en signant des lettres d'intention. Cette revue explore les avantages possibles pour un pays comme l'Irak qui a souffert pendant des années de la stagnation de son développement et de son éloignement des progrès technologiques ainsi que de la destruction des infrastructures.

Mots-clé : enseignement, français, coopération, stage, Irak.

\section{The impact of French cultural cooperation on language}

\begin{abstract}
This review is concerned with the cultural and scientific cooperation between Iraq and France. Through this cooperation the French government provided joint fellowship program in master and doctorate in various subjects. With regard to teaching French as a second foreign language the support included training courses (2-4 weeks) offered to French language teachers to help keep them abreast of the development of the language education sector. In addition, free curricula taught in the French language departments in five Iraqi universities has also been provided through this cooperation. Recently, in October 2017 the French government affirmed their continued willingness and commitment to participate in the rehabilitation of higher education in general and specifically revitalization of linguistic centers by signing a Goodwill Memorandum. This review, explores possible benefits to a country like Iraq, which has suffered for years from being away from development and advancement as well as the destruction of infrastructure..
\end{abstract}

Indexing terms/Keywords: French languge, cooperation, training, Iraq.

Subject Classification: French language, teaching French.

Type (Method/Approach): Mixed methods, case study; Historical Inquiry.

\section{Introduction}

Dans un pays comme l'Irak où la reconstruction est une tâche immense, loin d'être simple, « un pays mis à mal par des années de violence, le secteur éducatif doit faire face à la déscolarisation de milliers de jeunes et à la progression de l'illettrisme, plus particulièrement dans les zones rurales »(OIF, 2014 : 222), la réforme et la modernisation doivent viser tous les domaines aussi bien scientifiques que ceux qui relèvent des Sciences humaines; le système éducatif et les méthodes d'enseignement en général et celles du français en particulier 
doivent être revus en profondeur et bénéficier à leur tour des considérations d'une restructuration. Beacco(2009) montre que «la réorganisation des cursus des départements de français dans les universités irakiennes est une réponse nécessaire au nouveau contexte national et global ». Une telle réorganisation accompagnerait la politique linguistique du pays qui identifie de plus en plus des besoins d'apprentissages linguistiques nationaux en langues européennes et qui permettrait de faire évoluer les pratiques de l'enseignement du FLE en Irak, inadaptées au public.

Les systèmes éducatifs comme le souligne Davidenkoff (2014) « sont probablement aujourd'hui dans la même situation que l'industrie face à l'émergence de l'ordinateur personnel au milieu des années 70 ou les médias face à l'explosion du Web à la fin des années 90 ». Ils doivent donc s'adapter aux évolutions d'une époque et se développer en tenant compte des caractéristiques du public visé. C'est pourquoi il est nécessaire de connaître le besoin et l'attente de ce public, à la manière de lire le questionnement de Serres (2011) : "Avant d'enseigner quoi que ce soit à qui que ce soit, au moins faut-il le connaître. Qui se présente, aujourd'hui, à l'école, au collège, au lycée, à l'université ? ». Auparavant, le système éducatif irakien était considéré comme l'un des meilleurs de la région, mais est-ce qu'il l'est toujours ? Le pays a subi des guerres successives et un embargo depuis des années 90. Durant toutes ces années, il a été coupé du monde extérieur et de la communauté scientifique et de ses évolutions. Donc, les nécessités de reconstruction et de développement du pays sont très importantes et doivent toucher tous les domaines. Une telle reconstruction, un tel développement et une telle réhabilitation des infrastructures exigent un effort national et un soutien mondial.

\section{Présence et activités du service culturel français en Irak}

Le Centre culturel français (CCF) remplacé par l'Institut français en Irak (Ifi), le 11 février 2012, est le seul et le premier centre culturel étranger qui a rouvert ses portes en 1997 après les évènements de 1990 suite à l'entrée de l'armée irakienne au Koweït. L'Institut dont le but est de proposer des cours, formations et examens de français à un public aussi large que possible, il est accrédité pour faire passer et délivrer différentes certifications internationales, comme: le DELF, le DALF ou le TCF. Cet Institut est le lieu où se rendent les intéressés à la langue et la culture françaises.

En 1988, le Centre Culturel de Français à Bagdad commençait à fournir des cassettes vidéo au département de français à l'université de Mossoul pour donner l'occasion d'apprendre la langue française aux étudiants du département de même qu'à ceux qui étudiaient aux départements d'anglais et de traduction. Cette initiative permettait aux francophones d'assister aux séances de projection pour améliorer leur niveau en langue. L'année suivante en 1989, la semaine culturelle du cinéma français s'est tenue à la même université et des films français y ont été diffusés au public intéressé qui a apprécié cette action culturelle.

La présence du service culturel de l'Ambassade de France en Irak est marquée par les activités qu'il effectue régulièrement. Des dons et des aides recouvrent plusieurs domaines tels que linguistique, culturel, technique et économique. Comme l'Institut français à Bagdad, I'Institut français à Erbil, qui est inauguré en 2009, mène des activités variées. Les deux instituts préparent aux examens du DELF et DALF. Des cours de formation en langue y ont lieu régulièrement. Des expositions d'art et des activités culturelles invitent le public à s'y rendre. En ce qui concerne le domaine de l'Enseignement supérieur :

"Deux programmes de bourses à coût partagé s'adressant à un public qualifié (professeurs, fonctionnaires, chercheurs) ont été mis en place avec les autorités centrales et avec le gouvernement régional kurde. Ils permettent l'envoi en France de plusieurs dizaines de boursiers irakiens chaque année, qui suivent des cours de niveau master ou doctorat à l'issue d'une année de formation linguistique. En dépit du contexte sécuritaire, un soutien aux partenariats interuniversitaires continue à être apporté dans des disciplines prioritaires (mathématiques, informatique, médecine, droit,...) et la signature d'accords encouragée ». (OIF, ibid. $2014: 222$ )

Dans les années 80 et 90, un petit nombre des bacheliers se rendent en France pour faire des études supérieures c'est pourquoi, dans les universités irakiennes, nous ne comptons pas beaucoup de diplômés (master ou doctorat) 
formés en France. Selon la convention signée en 2009 entre l'Irak et La France, des centaines d'enseignants et de fonctionnaires irakiens ont participé à partir de 2011 au programme de bourses à coût partagé entre les deux pays qui permet de se former en master et doctorat.

Parmi ses missions, le conseiller français de coopération et d'action culturelle effectue des visites dans les universités irakiennes. Les entretiens et les discussions, avec les dirigeants, le corps enseignant et les étudiants des départements de français portent sur les moyens qui permettent de développer la coopération culturelle et linguistique entre l'Ambassade de France et les universités concernées. À titre d'exemple, au cours du mois de juin 2017, le $\operatorname{COCAC}^{1}$ a réalisé deux visites. D'une part, il a visité l'école Danielle Mitterrand de Soulaymanieh qui a été inaugurée en 2009 où le dispositif culturel et éducatif français est présent. Pour encourager les élèves, il a remis les attestations de réussite au diplôme du DELF prim aux lauréats de l'école qui ont réussi les examens (Ambassade de France, 2017c). D'autre part, il a pu se rendre à l'université de Soulaymanieh. Il a discuté avec les responsables de l'université des moyens de développer la coopération et considéré la volonté du président de l'université de créer un département de français au sein de cet établissement à condition toutefois d'avoir un soutien de la part de I'Ambassade de France.

Au cours du mois de février 2017, le COCAC s'est rendu dans les universités de Bagdad et Mustansirya où il a eu des échanges fructueux avec leurs responsables. Les moyens de développer la coopération entre l'Ambassade de France et ces deux universités ont été traités. Il a également rencontré le corps enseignant ainsi que les étudiants des départements du français de ces deux universités qu'il a félicités pour leur engagement et leur intérêt dans l'apprentissage de la langue et la culture françaises (Ambassade de France, 2017b). Ces rencontres régulières montrent l'engagement de la France pour le développement de l'enseignement du français en Irak. Le COCAC a annoncé que l'Institut français d'Irak a repris le travail pour donner des cours et former les intéressés en langue française.

\section{Formation et stages}

Le service de coopération et d'action culturelle assure régulièrement des stages de formation pour les enseignants irakiens du français. Ces stages dont la durée varie de deux semaines à un mois ont lieu en France dans les centres de langues. Ils peuvent être proposés localement à Abou Dhabi. Mais d'autres spécialités peuvent en profiter, à titre d'exemple, cinq archéologues irakiens ont participé, du 2 au 16 décembre 2017, à la formation de l'archéologie régionale et à la restitution des espaces anciens. Cette formation (Ambassade de France, 2017d) a été organisée conjointement par Archaïos et ses partenaires scientifiques que sont le Muséum National d'Histoire Naturelle de Paris, l'École Doctorale de l'université de Paris 1, le CNRS avec I'UMR 7041 de Nanterre.

Au cours du mois d'octobre 2017, des formations intensives (Ambassade de France, 2017e) dont la durée est de deux semaines ont été organisées à Vichy (sur l'innovation pédagogique), à Nantes (BELC, sur la didactique du français langue étrangère - FLE) et à Arras (sur la didactique du FLE/ FOS/FOU). Ces formations ont permis aux treize enseignants de quatre universités irakiennes qui $\mathrm{y}$ ont participé d'être sensibilisés aux techniques professionnelles les plus actuelles, d'en acquérir une certaine maîtrise et de rencontrer par la même occasion des acteurs clés de la profession.

Une autre formation qui a regroupé vingt enseignants s'est déroulée en novembre 2016 à l'Institut français d'IrakAntenne d'Erbil. Cette formation portait sur la thématique "Construire et intégrer les cours de FOS (Français sur Objectifs Spécifiques), une branche de l'enseignement du français langue étrangère (FLE), dans le cursus universitaire des départements de français" (Ambassade de France, 2016a). Le FOS concerne tous les domaines professionnels : affaires, tourisme, droit, médecine, sciences, relations internationales.

Dans le cadre d'une coopération culturelle et éducative avec le ministère de l'Éducation irakien et pour soutenir l'enseignement du français dans les écoles « Une centaine d'écoles, sur un total de 4.000 à 5.000 écoles, proposent aujourd'hui le français comme seconde langue étrangère » (Alassaf, $2016: 49$ ) et les départements de français, des actions ambitieuses ont été préparées par le SCAC ${ }^{2}$ afin de promouvoir l'enseignement du français. Les enseignants 
du français aux écoles peuvent aussi profiter de cette occasion en participant aux stages de formation en didactique du français langue étrangère (FLE).

Entre juillet 2015 et août 2016, une quarantaine d'enseignants ont assisté à quatre formations qui se sont déroulées au CAVILAM de Vichy. L'objectif était d'évaluer le niveau linguistique de ces participants par des pratiques d'enseignement du FLE. Les stages étaient consacrés « à une réflexion autour du changement de méthode en présentant aux stagiaires les manuels spécialisés les plus récents, et à l'utilisation et à l'adaptation de la méthode plébiscitée par les stagiaires lors de la précédente formation » (Ambassade de France, 2016b).

\section{Propositions du manuel}

Puren (1988 : 17) définit la méthode comme étant « l'ensemble des procédés et des techniques dont le but est de susciter chez l'apprenant un comportement ou une activité déterminée ». Depuis que l'enseignement du français a trouvé sa place dans le système éducatif irakien dans les années 60 , ses manuels et ses méthodes n'ont pas été régulièrement mis à jour. Nous remarquons par exemple qu'il y a plus de dix ans de décalage entre la date d'une parution et son application. Ni aux universités ni aux écoles, les enseignants irakiens ne restent libres de choisir le manuel sur lequel ils peuvent s'appuyer dans leur pratique. C'est d'ailleurs tout à fait contraire à ce que des didacticiens et pédagogues recommandent comme Pothier le souligne :

« Dans leur cadre institutionnel, les enseignants de FLE jouissaient d'une liberté quasi totale pour le choix des contenus, des méthodes ou des manuels. Ni instructions officielles, ni programme imposé dans la plupart des cas ; si des objectifs étaient donnés, les moyens pour les atteindre restaient du ressort de chaque enseignant, en négociation, pour un certain nombre d'entre eux, avec les apprenants concernés; cette liberté et cette responsabilité ont constitué un facteur important de créativité didactique » (Pothier, $2003: 70$ ).

Les enseignants se trouvent pris dans l'étau de contenus ou activités qu'ils estiment être loin des centres d'intérêt de leurs élèves. Cela démontre en quelque sorte les défauts possibles que peuvent avoir les manuels Blanchet (2006 : 12) indique que les manuels « empêchent de travailler. Ils font des enseignants, et souvent des apprenants aussi, de simples exécutants, répétiteurs dépendants d'un outil, et non concepteurs d'un enseignement ou d'un apprentissage appropriés ». C'est pourquoi un certain nombre d'enseignants s'évertuent à surmonter cela. Ils préparent et impriment, sur papiers, leurs propres explications et construisent des exemples de sujets imposés par le manuel qu'ils appliquent. Ils pensent que ce moyen aide leurs élèves à mieux comprendre lorsque des sujets ne sont pas facilement présentés dans le manuel en question.

De même, nous ne pouvons pas négliger les qualités qu'un manuel comprend. Il peut fournir aux intéressés (enseignant et apprenant) " un référent commun, un cadre, un outil, mais aussi une méthode et une logistique pédagogiques clé-en-main. À l'époque des moyens techniques de plus en plus sophistiqués (bandes-sons, supports visuels...), et de l'urgence dans laquelle la croissance rapide de la demande de formation met les enseignants, c'est là un atout non négligeable ». (Blanchet, ibid $2006: 12$ ).

En ce qui concerne les manuels utilisés dans les départements du français ou les écoles ou ceux des départements d'anglais en Irak, ils ne sont pas nombreux dès le commencement de l'enseignement de cette langue (Alassaf, 2016). Le premier manuel en ce qui concerne le français est Cours de langue et de civilisation françaises de Gaston Mauger, dont la date de la publication est 1953 de la librairie Hachette avec ses quatre volumes I, II, III et IV.

D'après le titre de ce manuel, il est clair que la langue et la civilisation y sont traitées. Il comprend des points grammaticaux, des textes de lecture, des révisions dont le but est double, celui de l'acquisition de la grammaire et celui du vocabulaire. Il est considéré comme " un cours moyen, une grammaire complète rédigée spécialement pour les étrangers et un livre de textes accompagnés de commentaires et dressant un tableau de la littérature et de la civilisation françaises ». (Mauger, Lamaison, et Hameau, 1958). Il est à noter que le volume I de ce manuel daté des années 50 est toujours utilisé dans les départements d'anglais des universités irakiennes pour le français en tant que deuxième langue étrangère. 
La France en direct de Jeanne Capelle, Guy Capelle, Francis Grand-Clément et Gilbert Quenelle, ouvrage publié en 1968 chez Hachette, est le deuxième manuel que les départements de français ont utilisé. Il comprend trois volumes dans lesquels des conversations, des situations, des textes et bien sûr de la grammaire y sont développés.

En 1997 et suite à une demande adressée aux ministères de l'Éducation et celui de l'Enseignement supérieur de la part du service culturel à l'Ambassade de France en Irak, un autre manuel a été proposé aux écoles et universités irakiennes. Il s'agit des deux volumes du Le Nouveau Sans Frontières de Philippe Dominique, Jacky Girardet, Michèle Verdelhan et Michel Verdelhan, manuel paru en 1987 de la maison d'édition CLE International.

Nous pouvons mentionner que les deux manuels cités ci-dessus (La France en direct et Le Nouveau Sans Frontières) étaient enseignés en même temps dans les deux niveaux scolaire et universitaire. D'une part, le volume I de chaque manuel est enseigné en première année de licence, d'autre part le même manuel est divisé en trois parties afin d'enseigner chaque partie dans une des trois années du collège. Tandis que le second volume de chaque manuel est quant à lui enseigné en deuxième année de licence. Il est également divisé en trois parties pour que les trois classes du lycée puissent en apprendre une partie par classe.

Le service culturel français tente toujours de renouveler les manuels et les supports employés dans le contexte irakien et c'est pourquoi il a organisé en 2008 et 2009 des séminaires afin de proposer un nouveau manuel. Le directeur du CAVILAM, les auteurs du manuel Alors ? (publié chez Didier en 2006) Jean-Claude Beacco et Marcella Di Giura, le conseiller culturel et 140 des enseignants de français d'universités et d'écoles y ont assisté (Wolff, 2010 : 114). Enfin, et pour la rentrée 2009, l'application du volume I du manuel Alors ?est entrée en vigueur en première année aux départements de français. L'année 2010 a vu l'utilisation du deuxième volume tandis que l'année suivante le volume III fut employé avec les étudiants de la troisième année.

Au CAVILAM durant le mois d'août 2016, des représentants du ministère de l'Éducation et du ministère de I'Enseignement supérieur irakiens furent invités à participer au stage consacré au choix d'un nouveau manuel ayant pour titre Kiosque 1 (de Fabienne Gallon, Adeline Gaudel, Céline Himber, Charlotte Rastello) paru en 2007. Diverses discussions ont été menées dans les moindres détails avec Adeline Gaudel, l'une des auteurs du manuel qui a accepté de faire des modifications pour le rendre adapté aux élèves irakiens. Nous avons réalisé des rencontres avec les participants et recueilli leur avis et nous avons également envoyé un courriel au directeur du CAVILAM pour savoir si ce livre pouvait remplacer celui utilisé pour l'enseignement jusqu'à aujourd'hui qui est Le nouveau sans frontières. Nous lisons ci-dessous sa réponse à notre courriel :

" Cher Collègue,

Merci pour votre courriel et votre intérêt pour notre travail et le projet d'introduction d'un manuel en Irak pour l'enseignement du français. Le choix définitif n'a toujours pas été validé par le ministère de l'Éducation. Le manuel pressenti actuellement est Kiosque publié aux éditions Hachette. Il est cependant possible que le choix se porte sur un autre manuel en cours d'évaluation. Bien cordialement. Michel Boiron $»^{3}$.

Bien que ce manuel soit basé sur les critères et recommandations du CECR, les stagiaires ne trouvent pas son contenu applicable dans le contexte irakien. La réponse du directeur du CAVILAM affirme qu'ils ne sont pas parvenus à se mettre d'accord pour l'appliquer. Ils souhaitent garder le manuel actuel auquel tous les enseignants irakiens en langue française sont habitués parce qu'ils l'enseignent depuis vingt ans. Beaucoup de critères doivent être pris en compte lors du choix d'une méthode. Blanchet résume ces critères d'analyse didactique d'une méthode de langue comme suit :

\footnotetext{
« * titre

* auteur(s), qualifications annoncées

* éditeur et partenaires

* année de publication

* matériels
} 
* tables des matières et autres plans

* introduction/commentaires pédagogiques

* public(s) et objectifs visé(s)

* structure-type d'une "unité"

* types de contenus communicatifs, culturels, langagiers

et pédagogiques (activités, exercices...) ». (Blanchet, ibid, $2006: 7-8$ )

Ces critères ne furent pas traités en détail lors de la réunion du comité sectoriel pour le curriculum des facultés de Lettres dans les universités irakiennes tenue à Bagdad au milieu du mois de novembre 2017. Les chefs des départements de langues ont discuté et critiqué les programmes d'enseignement actuel, mais ne voulaient ni les changer ni les moderniser. Un tel changement exigeant, bien sûr, des ressources, des démarches et du temps pour qu'il puisse voir le jour a contraint au maintien de ce qui existait actuellement sur le terrain.

L'Ambassade de France ne manque pas de saisir toutes les occasions pour collaborer avec le ministère de l'Éducation et le ministère de l'Enseignement supérieur irakiens. Le 5 fév. 2017, l'Ambassadeur de France a reçu le Ministre de l'Éducation ayant pour but d'entreprendre une discussion sur l'enseignement du français et la coopération éducative franco-irakienne. L'un et l'autre se sont entretenus sur les formations que l'Ambassade organise chaque année en France pour former les enseignants de français du secondaire ainsi que du supérieur. Nous jugeons bon de citer les points abordés lors de cette rencontre :

«L'Ambassadeur a remis au Ministre un exemplaire de la méthode Génération, qui pourrait être utilisée dans le contexte culturel irakien et faire l'objet d'un don au profit des lycées irakiens. Il a aussi plaidé en faveur du maintien de la place du français dans le système éducatif irakien, et s'est inquiété de mesures récentes comme la réduction du nombre d'heures hebdomadaires d'enseignement du français dans les écoles, la suppression du département du français au sein du ministère de l'Education ou encore la transformation en option de l'apprentissage du français dans les écoles pilotes. Le Ministre a pour sa part ajouté qu'il relevait également des universités de soutenir l'enseignement du français en proposant des filières de spécialités en accord avec le marché du travail irakien » (La France en Irak, 2017a).

La proposition d'un autre manuel tel que Génération par l'Ambassadeur de France montre clairement que la France demeure toujours présente pour soutenir l'enseignement du français en Irak. Elle essaie également de proposer de nouvelles méthodes et de nouveaux manuels pouvant être adoptés dans le contexte irakien. Afin de choisir une méthode, des critères sont à respecter. Malvaux (1996:33) pose des questions du genre : " à quel public la méthode s'adresse-t-elle (aux enfants, aux adolescents ou aux adultes) ? [...] Quelle méthodologie préconise la méthode (est-ce une méthode audio-orale, audiovisuelle, une simulation globale, etc.) ? ». Ces points sont nécessaires à évoquer car ils orientent l'enseignant de la langue vers un apprentissage efficient à l'égard de ses élèves.

Le 19 mars 2017, le Ministre irakien de I'Enseignement supérieur et de la recherche scientifique a reçu l'Ambassadeur de France à Bagdad. Ils ont discuté de la coopération culturelle et scientifique. Leur entretien a porté sur l'amélioration du programme franco-irakien de bourses à coûts partagés selon lequel 100 personnes se rendent en France chaque année afin de poursuivre des études de master et doctorat. Le Ministre s'est notamment demandé si les médecins irakiens pouvaient effectuer un stage clinique dans des hôpitaux français à l'instar des enseignants du français.

\section{Soutien de la France à la réhabilitation}

David (2017) pose la question suivante: "L'Irak se relèvera-t-il, et comment, de décennies de dictature, d'une invasion américaine, d'une guerre civile et influences extérieures? ». Nous pouvons nous appuyer sur la déclaration du président de la République Française (Elysées, 2017) lors d'une rencontre avec le Premier Ministre Irakien : « Nos échanges ont permis justement de poser les bases d'un nouveau partenariat entre l'Irak et la France. Nos deux pays ont des liens anciens, solides, et nous avions, dans le passé, de très nombreuses coopérations avec l'Irak, dans tous les domaines ». Suite aux entretiens intensifs, nous constatons qu'il y a un engagement important dans les 
domaines culturels, universitaires et économiques. Des lettres d'intention sont signées entre les deux pays dont le but est de structurer une coopération en matière d'enseignement supérieur. Cette coopération permettra de réhabiliter l'université de Mossoul. Les deux parties ont discuté les moyens de coopération bilatérale et ont évoqué la volonté de la partie irakienne d'activer la coopération scientifique avec les universités françaises par le biais de programmes de bourses mutuelles. (Iraqi Ministery of Foreign Affairs, 2017).

À l'instar de cet engagement, au cours du mois de décembre 2017, l'Ambassadeur de France à Bagdad a reçu les présidents de l'université de Mossoul et de Ninive chacun à part. Ils ont évoqué les possibilités de relancer la coopération avec des universités françaises. L'Ambassadeur y a confirmé la décision de la France de financier la reconstruction et la réhabilitation des bâtiments universitaires. Actuellement, des procédures d'aménager un coin de la francophonie ${ }^{4}$ au sein de l'université de Mossoul est en cours. Ce coin sera le point d'un nouveau départ pour la coopération culturelle et scientifique entre cette université et les universités francophones.

Côté enseignants du français, cet engagement représente l'espoir d'un processus d'entrée en compétences nécessaires et de méthodes d'enseignement pour qu'ils choisissent les stratégies convenant à leur environnement et à la position dans laquelle ils travaillent (établissements scolaires et universitaires). À noter que (Alassaf, 2016) « la problématique des TIC dans le domaine de l'éducation et de l'enseignement est marquée par des contraintes. Les équipements informatiques et multimédias ne sont ni suffisants ni pertinents et ne proposent pas de modalités innovantes ni de développement numérique ». Les ressources humaines doivent également se lancer dans ces modalités pour prendre en charge l'innovation souhaitée.

C'est pourquoi il est important de considérer l'importance de cet engagement et ce soutien permettant par la suite de revoir la réhabilitation des bâtiments universitaires et d'obtenir des équipements, des laboratoires, des infrastructures qui manquent dans les deux universités à cause de la situation liée aux événements vécus depuis juin 2014 jusqu'à ce jour.

\section{Conclusion}

Dans ce travail, nous avons développé la présence et le soutien de l'action culturelle et linguistique qu'assurent l'Ambassade de France en Irak. Nous avons évoqué tout l'intérêt de l'enseignement du FLE face aux défis actuels et initié son débat pertinent.

Nous constatons que les facilités et l'aide que proposent les services culturels, économiques et linguistiques représentent des atouts majeurs pour favoriser l'apprentissage de la langue française au grand public et à en construire ainsi les ponts nécessaires aux échanges sociaux économiques et culturels.

Les discussions bilatérales entre la France et l'Irak concernant la question du réaménagement du programme franco-irakien de bourses universitaires à coût partagé et celle de la prise en compte des notes du français aux examens de fin d'études secondaires dans l'enseignement irakien sont à l'ordre du jour.

L'Institut français et les universités qui proposent les cours du FLE et FOS permettent d'accompagner les apprenants plus efficacement dans la préparation de leur projet professionnel en adaptant au mieux leur apprentissage du français au contexte de leur futur métier. La coopération peut à nouveau se développer en Irak et qu'elle est nécessaire pour relancer l'apprentissage de la langue française.

\section{Conflicts of Interest}

There are no conflicts of interest concerning this research study.

\section{Funding Statement}

The research was funded only by the author. 


\section{Références}

1. Alassaf, Y. Intégration des TIC dans l'enseignement/apprentissage du FLE en Irak: enjeux institutionnels, organisationnels et pédagogiques, 440 p. Thèse : didactique des langues : Lille III, 2016.

2. Ambassade de France. (2017a). La France en Irak. [En ligne] 07 fév. 2017. [réf. du 12 mars 2017]. Disponible sur: $\quad$ https://iq.ambafrance.org/Reunion-entre-MM-I-Ambassadeur-de-France-et-Mohamed-Iqbal-leministre-de-I. (URL vérifié 31 oct.2018).

3. Ambassade de France. (2017b). La France en Irak. Actualités culturelles, humanitaire et de coopération. Visite du Conseiller de coopération et d'action culturelle dans les universités de Bagdad et Mustansirya. [En ligne] 14 mai 2017. [réf. du 22 janvier 2018]. Disponible sur: https://iq.ambafrance.org/Visite-duConseiller-de-cooperation-et-d-action-culturelle-dans-les-Universites. (URL vérifié 31 oct.2018).

4. Ambassade de France. (2017c). La France en Irak. Actualités culturelles, humanitaire et de coopération. Visite du Conseiller de coopération et d'action culturelle à l'université de Soulaymanieh. [En ligne] 30 juillet 2017. [réf. du 22 janvier 2018]. Disponible sur: https://iq.ambafrance.org/Visite-du-Conseiller-decooperation-et-d-action-culturelle-a-I-universite. (URL vérifié le 31 oct.2018).

5. Ambassade de France. (2017d). La France en Irak. Actualités culturelles, humanitaire et de coopération. Formation de 5 archéologues irakiens à Paris. [En ligne] 31 décembre 2017. [réf. du 24 janvier 2018]. Disponible sur : https://iq.ambafrance.org/ Formation-de-5-archéologues-irakiens-à-Paris. (URL vérifié le 31 oct.2018).

6. Ambassade de France. $\left(2017^{\mathrm{e}}\right)$. La France en Irak. Actualités culturelles, humanitaire et de coopération. Stages de formation pour l'enseignant du français. [En ligne] 28 octobre 2017. [réf. du 24 janvier 2018]. Disponible sur: https://iq.ambafrance.org/Stages-de-formation-a-I-innovation-et-a-la-didactique-de-Ienseignement-du. . (URL vérifié le 31 oct.2018).

7. Ambassade de France. (2016a). La France en Irak. Actualités culturelles, humanitaire et de coopération. Un stage de formation à I'Institut français d'Irak-Antenne d'Erbil. [En ligne] 18 décembre 2016. [réf. du 24 janvier 2018]. Disponible sur: https://iq.ambafrance.org/Un-stage-de-formation-a-I-Institut-francais-dIrak-Antenne-d-Erbil. (URL vérifié le 31 oct.2018).

8. Ambassade de France. (2016b). La France en Irak. Actualités culturelles, humanitaire et de coopération. Formation en didactique du Français Langue Etrangère FLE destinée aux enseignants de langue française du ministère de l'éducation. [En ligne] 28 août 2016. [réf. du 24 janvier 2018]. Disponible sur: https://iq.ambafrance.org/Formation-en-didactique-du-Francais-Langue-Etrangere-FLE-destinee-aux. (URL vérifié le 31 oct.2018).

9. Blanchet, Ph. (2006). Méthodes et méthodologies pour l'enseignement des langues étrangères (l'exemple du français langue étrangère). Cours SUED/CREA filmé de Ph. Blanchet. 2005-2006. UED de didactique des langues. Licences 3. Rennes.

10. Beacco, J-C. (2009). Rénovation des cursus des Départements de français des universités irakiennes. Compte rendu des entretiens et propositions (Séminaire tenu à Paris du 8 au 12 décembre 2008).

11. Boiron, M. (2016). Le manuel Kiosque 1. Une réponse du directeur de CAVILAM à Vichy le, 23 août 2016 à notre courriel daté du 19 août 2016.

12. David, D. (2017). Politique étrangère. Revue trimestrielle de l'Ifri, numéro d'hiver 2017-2018.

https://www.ifri.org/fr/espace-media/videos/lirak-apres-daech-politique-etrangere-hiver-2017-2018. 
13. Davidenkoff, E. (2014). Le tsunami numérique : éducation, tout va changer ! Êtes-vous prêts ? Paris, France : Stock.

14. Elysées. (2017). Déclaration conjointe du président de la république Emmanuel Macron et du premier ministre irakien. [En ligne] 5 octobre 2017. [réf. du 25 janvier 2018]. Disponible sur :

http://www.elysee.fr/declarations/article/declaration-conjointe-du-president-de-la-republique-emmanuelmacron-et-du-premier-ministre-irakien/. (URL vérifié le 31 oct.2018).

15. Iraqi Ministery of Foreign Affairs. (2017). Signature des lettres de "déclaration d'intention" entre l'Irak et la France. [En ligne] 11 octobre 2017. [réf. du 25 janvier 2018]. Disponible sur: http://mofamission.gov.iq/en/France\&article=14066. (URL vérifié le 31 oct.2018).

16. Malvaux, I. (1996). Une décision difficile : le choix d'une méthode. Le point sur le FLE. Français 2000, numéro double, juin 1996. Bulletin trimestriel juin 1996.

17. Mauger, G, Lamaison, J. et Hameau, A-M. (1958). Cours de langue et de civilisation françaises. Paris, France: Hachette.

18. OIF. (2014). Rapport la langue française dans le monde. La langue française. [En ligne] 2014, [réf. du 12 janvier 2018]. Disponible sur : https://www.francophonie.org/Rapport-la-langue-francaise-dans.html. (URL vérifié le 31 oct.2018).

19. Pothier, M. (2003). Multimédias, dispositifs d'apprentissage et acquisition des langues. Collection Autoformation et enseignement multimédia 11. Paris : Ophrys.

20. Puren, C. (1988). Histoire des méthodologies de l'enseignement des langues. Didactique des langues étrangères 13. Paris: Clé international : [diff. Nathan].

21. Serres, M. (2011). « Petite Poucette. Les nouveaux défis de l'Éducation ». [En ligne] 1 mars 2011, [réf. du 25 janvier 2018]. Disponible sur : http://www.academie-francaise.fr/petite-poucette-les-nouveaux-defis-deleducation. (URL vérifié le 31 oct. 2018).

22. Wolff, A. (2010). La langue française dans le monde 2010. Éditions Nathan. La Francophonie. Paris: OIF. [En ligne] 2010, [réf. du 25 janvier 2018]. Disponible sur :

www.francophonie.org/IMG/pdf/langue_francaise_monde_integral.pdf. (URL vérifié le 31 oct.2018).

$\left[{ }^{1}\right]$ Le conseiller de coopération et d'action culturelle.

$\left.{ }^{2}\right]$ Service de coopération et d'action culturelle.

[3]Une réponse officielle de la part de Michel Boiron., directeur du CAVILAM à Vichy. 23 août 2016.

$\left[{ }^{4}\right]$ Nous avons recueilli ces informations lors des rencontres et discussions, au cours du mois de janvier 2018, avec notre collègue qui est désigné comme responsable de ce nouveau projet qui doit voir le jour pendant 2018. 